\title{
An Innovative Direct NF-FF Transformation Technique with Helicoidal Scanning
}

\author{
Francesco D’Agostino, Flaminio Ferrara, Claudio Gennarelli, \\ Rocco Guerriero, and Massimo Migliozzi
}

\author{
Department of Electronic and Computer Engineering, University of Salerno, via Ponte Don Melillo, \\ I-84084 Fisciano (Salerno), Italy \\ Correspondence should be addressed to Claudio Gennarelli, gennar@diiie.unisa.it \\ Received 14 September 2011; Accepted 16 February 2012 \\ Academic Editor: Lars Jacob Foged
}

Copyright () 2012 Francesco D’Agostino et al. This is an open access article distributed under the Creative Commons Attribution License, which permits unrestricted use, distribution, and reproduction in any medium, provided the original work is properly cited.

A direct near-field-far-field transformation with helicoidal scanning is developed. It is based on the nonredundant sampling representation of electromagnetic fields and uses a spherical antenna modelling to determine the number of helix turns. Moreover, the number of voltage samples on each of them is fixed by the maximum transverse dimension of the antenna, both to simplify the mechanical scanning and to reduce the computational effort. This technique allows the evaluation of the antenna far field directly from a minimum set of near-field data without interpolating them. Although the number of near-field data employed by the developed technique is slightly increased with respect to that required by rigorously applying the nonredundant sampling representation on the helix, it is still remarkably smaller than that needed by the standard near-field-far-field transformation with cylindrical scanning. The effectiveness of the technique is assessed by numerical and experimental results.

\section{Introduction}

The techniques for the reconstruction of antenna radiation patterns from near-field (NF) measurements have been widely investigated and used for applications ranging from cellular phone antennas to large-phased arrays and complex multibeam communication satellite antennas [1-5]. They have been proved to be efficient and attractive alternatives to conventional far-field (FF) and compact range measurements. In addition, the NF measurements have the advantage to be performed in a controlled environment, such as an anechoic chamber, thus overcoming those drawbacks due to weather conditions, electromagnetic (EM) interference, and so forth, which cannot be eliminated in FF outdoor measurements. In the recent years, the researchers interested in the antenna NF measurements and in the related NF-FF transformation techniques have spent many efforts to reduce the time required for the acquisition of the NF data. As a matter of fact, this time is currently very much greater than that needed to carry out the corresponding NF-FF transformation. In this context, a significant reduction of the number of required NF data (and, as a consequence, of the measurement time) has been obtained for all the conventional scannings [6-14] by applying the theoretical results on the nonredundant sampling representations of EM fields [15]. In particular, optimal sampling interpolation (OSI) formulas of central type [16] have been employed to efficiently recover the data required by the corresponding NF-FF transformation from the knowledge of the acquired nonredundant ones.

A more convenient way of reducing the measurement time is the use of innovative spiral scanning techniques. They have been implemented, as suggested by Yaccarino et al. in [17], by means of continuous and synchronized movements of the positioning systems of the probe and antenna under test (AUT), and are based on the aforementioned nonredundant sampling representations and OSI expansions. In particular, NF-FF transformations using the helicoidal scanning [18-20], the planar [20,21] and spherical $[20,22]$ spiral scannings have been developed, by considering the AUT as enclosed in the smallest sphere able to contain it. 
Then, more effective AUT modellings, that allow a further reduction of the required NF data in the case of elongated or quasiplanar antennas, have been adopted in [23-27] by properly employing the unified theory of spiral scans for nonspherical antennas [28]. These modellings allow one to consider measurement cylinders (planes) with a radius (distance) smaller than one half the AUT maximum size, thus reducing the error related to the truncation of the scanning zone. In all the cases, the NF data needed by the corresponding NF-FF transformation can be reconstructed by interpolating the nonredundant ones acquired on the spiral.

A probe uncompensated NF-FF transformation technique with planar spiral scanning has been proposed in [29], to efficiently evaluate the antenna far field directly from the acquired NF data. It uses the convolution property of the radiation integral and the fast Fourier transform (FFT) algorithm to determine the antenna far-field pattern without requiring any interpolation step. However, since such an approach does not exploit the nonredundant representations of EM fields, it needs a useless large amount of measurements. This comment holds also for the NF-FF transformation technique with helicoidal scanning proposed by the same authors in [30]. On the contrary, a direct NF-FF transformation with cylindrical scanning, based on the aforementioned nonredundant sampling representations, has been developed in [10]. It allows the evaluation of the antenna far field in any cut plane directly from the nonredundant NF data without interpolating them.

In this paper, the direct NF-FF transformation with cylindrical scanning [10] has been extended to the helicoidal one [18-20]. To this end, the approach in [10] has been reviewed in order to match the advantages of the direct cylindrical NF-FF transformation with those own of the fast helicoidal scanning. In particular, the number of helix turns is determined by the nonredundant sampling representation along a generatrix which makes use of the spherical AUT modelling, whereas the voltage samples on each of them is fixed by the AUT maximum transverse dimension.

\section{Nonredundant Sampling Representations on a Cylinder from Helicoidal Samples}

The nonredundant sampling representation of the probe voltage on a cylinder from NF data acquired along a helix, when adopting a spherical AUT modelling, is summarized in the following.

Let us consider a nondirective probe which scans a helix with constant angular step lying on a cylinder of radius $d$ surrounding the AUT (see Figure 1) and adopt the spherical coordinate system $(r, \vartheta, \varphi)$ for denoting the observation point $P$ in the NF region. Since the voltage $V$ measured by this kind of probe has the same effective spatial bandwidth of the field [7], the theoretical results on the nonredundant representation of EM fields [15] can be applied to such a voltage. Accordingly, if the AUT is enclosed in a sphere of radius $a$ (AUT ball) and the helix is described by a proper

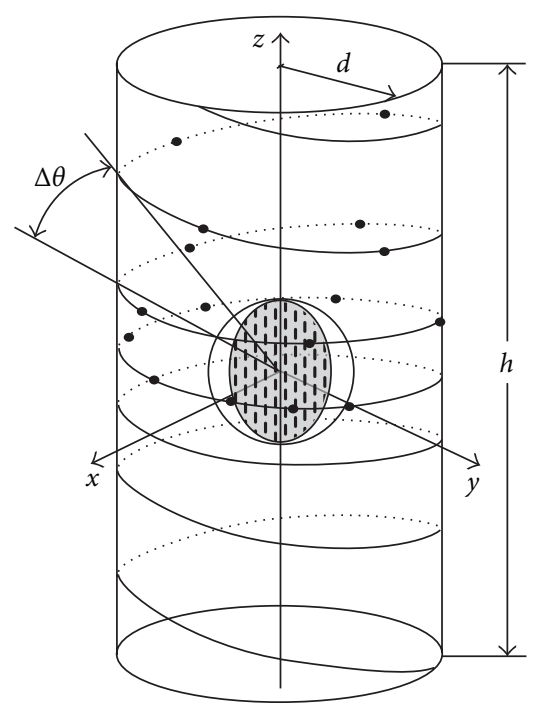

Figure 1: Helicoidal scanning.

analytical parameterization $\underline{r}=\underline{r}(\xi)$, the probe "reduced voltage"

$$
\widetilde{V}(\xi)=V(\xi) e^{\mathrm{j} \gamma(\xi)}
$$

can be closely approximated by a spatially band limited function [15], $\gamma(\xi)$ being a phase function to be determined. The related band limitation error becomes negligible as the bandwidth exceeds a critical value $W_{\xi}[15]$, so that it can be effectively controlled by choosing a bandwidth equal to $\chi^{\prime} W_{\xi}$, wherein the excess bandwidth factor $\chi^{\prime}$ is slightly greater than unity for an electrically large AUT.

To obtain a sampling representation of the voltage on the cylinder from its nonredundant samples collected along a helix with constant angular step, it is necessary (a) to develop a nonredundant voltage representation on the helix; (b) to choose the helix step equal to the sample spacing needed to interpolate the voltage along a cylinder generatrix.

The parametric equations of the helix, when imposing its passage through a fixed point $Q_{0}$ of the generatrix at $\varphi=0$, are $x=d \cos \left(\phi-\phi_{i}\right), y=d \sin \left(\phi-\phi_{i}\right), z=d \cot \theta$, where $\phi$ is the angular parameter describing the helix, $\phi_{i}$ is the value of $\phi$ at $Q_{0}$, and $\theta=k \phi$. The parameter $k$ is such that the angular step, determined by the consecutive intersections $Q(\phi)$ and $Q(\phi+2 \pi)$ with a generatrix, is $\Delta \theta=2 \pi k$. It is worth noting that the helix can be obtained by radially projecting on the measurement cylinder the spiral wrapping the AUT sphere with the same angular step.

As shown in $[19,20]$, a nonredundant sampling representation of the voltage on the helix can be obtained by using the following expressions for the optimal phase function and parameterization:

$$
\begin{aligned}
& \gamma=\beta \int_{0}^{r} \sqrt{1-\frac{a^{2}}{r^{\prime 2}}} \mathrm{~d} r^{\prime}=\beta \sqrt{r^{2}-a^{2}}-\beta a \cos ^{-1}\left(\frac{a}{r}\right), \\
& \xi=\frac{\beta a}{W_{\xi}} \int_{0}^{\phi} \sqrt{k^{2}+\sin ^{2} k \phi^{\prime}} \mathrm{d} \phi^{\prime},
\end{aligned}
$$

where $\beta$ is the wavenumber. 
Since the elevation step of the helix must be equal to the sample spacing required for the interpolation along a generatrix, then, according to $[19,20], \Delta \theta=2 \pi /\left(2 N^{\prime \prime}+1\right)$, with $N^{\prime \prime}=\operatorname{Int}\left(\chi N^{\prime}\right)+1$ and $N^{\prime}=\operatorname{Int}\left(\chi^{\prime} \beta a\right)+1, \chi>$ 1 being an oversampling factor controlling the truncation error $[15,16]$ and $\operatorname{Int}(x)$ denoting the integer part of $x$. It can be easily verified that $k=1 /\left(2 N^{\prime \prime}+1\right)$.

According to (3), $\xi$ is proportional to the curvilinear abscissa along the spiral wrapping the sphere modelling the source. Since such a spiral is a closed curve, it is convenient to choose the bandwidth $W_{\xi}$ such that $\xi$ covers a $2 \pi$ range when the whole curve on the sphere is described. As a consequence,

$$
W_{\xi}=\frac{\beta a}{\pi} \int_{0}^{\left(2 N^{\prime \prime}+1\right) \pi} \sqrt{k^{2}+\sin ^{2} k \phi^{\prime}} \mathrm{d} \phi^{\prime}
$$

According to these results, the OSI formula of central type to reconstruct the voltage at any point $Q$ of the helix is [20]:

$$
\tilde{V}(\xi)=\sum_{m=m_{0}-p+1}^{m_{0}+p} \tilde{V}\left(\xi_{m}\right) \Omega_{M}\left(\xi-\xi_{m}\right) D_{M^{\prime \prime}}\left(\xi-\xi_{m}\right)
$$

where $m_{0}=\operatorname{Int}\left[\left(\xi-\xi\left(\phi_{i}\right)\right) / \Delta \xi\right]$ is the index of the sample nearest (on the left) to $Q, 2 p$ is the number of retained samples $\tilde{V}\left(\xi_{m}\right)$, and

$$
\xi_{m}=\xi\left(\phi_{i}\right)+m \Delta \xi=\xi\left(\phi_{i}\right)+\frac{2 \pi m}{\left(2 M^{\prime \prime}+1\right)}
$$

with $M^{\prime \prime}=\operatorname{Int}\left(\chi M^{\prime}\right)+1$ and $M^{\prime}=\operatorname{Int}\left(\chi^{\prime} W_{\xi}\right)+1$. Moreover,

$$
\begin{aligned}
D_{M^{\prime \prime}}(\xi) & =\frac{\sin \left(\left(2 M^{\prime \prime}+1\right) \xi / 2\right)}{\left(2 M^{\prime \prime}+1\right) \sin (\xi / 2)}, \\
\Omega_{M}(\xi) & =\frac{T_{M}\left[-1+2(\cos (\xi / 2) / \cos (\bar{\xi} / 2))^{2}\right]}{T_{M}\left[-1+2 / \cos ^{2}(\bar{\xi} / 2)\right]}
\end{aligned}
$$

are the Dirichlet and Tschebyscheff sampling functions, wherein $T_{M}(\xi)$ is the Tschebyscheff polynomial of degree $M=M^{\prime \prime}-M^{\prime}$ and $\bar{\xi}=p \Delta \xi$.

The OSI formula (5) can be used to evaluate the "intermediate samples," namely, the voltage values at the intersection points between the helix and the generatrix passing through the observation point $P$. Once these samples have been evaluated, the voltage at $P$ can be reconstructed via the following OSI expansion:

$$
\tilde{V}(\vartheta, \varphi)=\sum_{n=n_{0}-q+1}^{n_{0}+q} \tilde{V}\left(\vartheta_{n}\right) \Omega_{N}\left(\vartheta-\vartheta_{n}\right) D_{N^{\prime \prime}}\left(\vartheta-\vartheta_{n}\right)
$$

where $N=N^{\prime \prime}-N^{\prime}, n_{0}=\operatorname{Int}\left[\left(\vartheta-\vartheta_{0}\right) / \Delta \vartheta\right], 2 q$ is the number of the retained intermediate samples $\widetilde{V}\left(\vartheta_{n}\right)$, and

$$
\vartheta_{n}=\vartheta_{n}(\varphi)=\vartheta\left(\phi_{i}\right)+k \varphi+n \Delta \vartheta=\vartheta_{0}+n \Delta \vartheta
$$

The described two-dimensional OSI algorithm can be properly applied to recover the NF data required by the NFFF transformation technique [31] or [32].

\section{Standard NF-FF Transformation with Cylindrical Scanning}

For reader's convenience, the key steps of the standard probe compensated NF-FF transformation technique with cylindrical scanning [31] are reported. According to such a technique, the modal coefficients $a_{v}$ and $b_{v}$ of the cylindrical wave expansion of the field radiated by the AUT are related to (i) the two-dimensional Fourier transforms $I_{\nu}^{1}$ and $I_{\nu}^{2}$ of the output voltage of the probe for two independent sets of measurements (the probe is rotated $90^{\circ}$ about its longitudinal axis in the second set); (ii) the coefficients $c_{m}, d_{m}$, and $c_{m}^{\prime}, d_{m}^{\prime}$ of the cylindrical wave expansion of the field radiated by the probe and the rotated probe, respectively, when used as transmitting antennas. The key relations are

$$
\begin{aligned}
a_{\nu}(\eta)= & \frac{\beta^{2}}{\Lambda^{2} \Delta_{\nu}(\eta)}\left[I_{\nu}^{1}(\eta) \sum_{m=-\infty}^{\infty} d_{m}^{\prime}(-\eta) H_{\nu+m}^{(2)}(\Lambda d)\right. \\
& \left.-I_{\nu}^{2}(\eta) \sum_{m=-\infty}^{\infty} d_{m}(-\eta) H_{\nu+m}^{(2)}(\Lambda d)\right], \\
b_{\nu}(\eta)= & \frac{\beta^{2}}{\Lambda^{2} \Delta_{\nu}(\eta)}\left[I_{\nu}^{2}(\eta) \sum_{m=-\infty}^{\infty} c_{m}(-\eta) H_{\nu+m}^{(2)}(\Lambda d)\right. \\
& \left.-I_{\nu}^{1}(\eta) \sum_{m=-\infty}^{\infty} c_{m}^{\prime}(-\eta) H_{\nu+m}^{(2)}(\Lambda d)\right], \\
I_{\nu}^{1,2}(\eta)= & \int_{-\infty}^{\infty} \int_{0}^{2 \pi} V^{1,2}(\varphi, z) e^{-j \nu \varphi} e^{j \eta z} \mathrm{~d} \varphi \mathrm{d} z, \\
\Delta_{v}(\eta)= & \sum_{m=-\infty}^{\infty} c_{m}(-\eta) H_{\nu+m}^{(2)}(\Lambda d) \\
& \times \sum_{m=-\infty}^{\infty} d_{m}^{\prime}(-\eta) H_{\nu+m}^{(2)}(\Lambda d) \\
& -\sum_{m=-\infty}^{\infty} c_{m}^{\prime}(-\eta) H_{\nu+m}^{(2)}(\Lambda d) \\
& \times \sum_{m=-\infty}^{\infty} d_{m}(-\eta) H_{\nu+m}^{(2)}(\Lambda d)
\end{aligned}
$$

where $\Lambda=\sqrt{\beta^{2}-\eta^{2}}, H_{\nu}^{(2)}(\cdot)$ is the Hankel function of second kind and order $v$, and $V^{1}, V^{2}$ represent the output voltage of the probe and the rotated probe at the point of cylindrical coordinates $(d, \varphi, z)$. 
Once the modal coefficients have been determined, the FF components of the electric field in the spherical coordinate system $(R, \Theta, \Phi)$, adopted to denote an observation point in the far-field region, can be evaluated by

$$
\begin{aligned}
E_{\Theta}(R, \Theta, \Phi) & =F_{\Theta}(\Theta, \Phi) \frac{e^{-j \beta R}}{R} \\
& =-j 2 \beta \frac{e^{-j \beta R}}{R} \sin \Theta \sum_{\nu=-\infty}^{\infty} j^{\nu} b_{\nu}(\beta \cos \Theta) e^{j \nu \Phi}, \\
E_{\Phi}(R, \Theta, \Phi) & =F_{\Phi}(\Theta, \Phi) \frac{e^{-j \beta R}}{R} \\
& =-2 \beta \frac{e^{-j \beta R}}{R} \sin \Theta \sum_{\nu=-\infty}^{\infty} j^{\nu} a_{\nu}(\beta \cos \Theta) e^{j \nu \Phi} .
\end{aligned}
$$

\section{Direct NF-FF Transformation Technique}

The here proposed approach for evaluating the antenna FF pattern directly from the acquired helicoidal samples is described in the following. In particular, such a NF-FF transformation, unlike that developed in $[18,20]$, does not explicitly employ the OSI expansions (5) and (8), since there is no need to interpolate the nonredundant acquired data for reconstructing those needed by the classical ones [31, 32].

The starting point is the evaluation of the twodimensional Fourier transforms of the probe output voltage in (11). By taking into account the OSI formula (8), we can rearrange the integrals (11) in the form:

$$
\begin{aligned}
I_{\nu}^{1,2}(\eta)= & \sum_{n \in N_{r}} \int_{0}^{2 \pi}\left\{\tilde{V}^{1,2}\left(\vartheta_{n}, \varphi\right) e^{-j \nu \varphi}\right. \\
& \left.\times \int_{-\infty}^{\infty} D_{N^{\prime \prime}}\left(\vartheta(z)-\vartheta_{n}\right) Q\left(\vartheta(z)-\vartheta_{n}\right) e^{-j \gamma(z)} e^{j \eta z} \mathrm{~d} z\right\} \mathrm{d} \varphi,
\end{aligned}
$$

where $N_{r}$ is the set of indexes of all considered NF turns, $\widetilde{V}^{1,2}\left(\vartheta_{n}, \varphi\right)$ are the reduced voltages samples at the intersection points between the generatrix at $\varphi$ and the scanning helix, and $Q=\Omega_{N}$, if $\left|\vartheta(z)-\vartheta_{n}\right| \leq q \Delta \vartheta$, or $Q=0$, otherwise. Let us first consider the integration over $z$. For any fixed $\varphi$, it results

$$
G_{n \eta}(\varphi)=\int_{z_{i}}^{z_{f}} D_{N^{\prime \prime}}\left(\vartheta(z)-\vartheta_{n}\right) \Omega_{N}\left(\vartheta(z)-\vartheta_{n}\right) e^{-j \gamma(z)} e^{j \eta z} \mathrm{~d} z,
$$

where $z_{i}=z\left(\vartheta_{n}+q \Delta \vartheta\right)$ and $z_{f}=z\left(\vartheta_{n}-q \Delta \vartheta\right)$. By taking into account (15), the relation (14) can be rewritten as follows:

$$
I_{\nu}^{1,2}(\eta)=\sum_{n \in N_{r}} \int_{0}^{2 \pi} \tilde{V}^{1,2}\left(\vartheta_{n}, \varphi\right) G_{n \eta}(\varphi) e^{-j \nu \varphi} \mathrm{d} \varphi .
$$

Such a relation involves an integration over $\varphi$ that must be solved numerically for any $n \in N_{r}$, by using, from time to time, the voltage samples on the considered helix turn, whose number varies, according to the nonredundant sampling representation on the helix (see Section 2), when moving from the central to the peripheral turns and does not ensure the convergence of the numerical integration. Moreover, the $G_{n \eta}(\varphi)$ values depend not only on the measurement cylinder and on the radius of the sphere enclosing the AUT, but also on the points at which the generatrix passing through $\varphi$ intersects the scanning helix. It can be easily recognized that integration over $\varphi$ can be efficiently solved by using the FFT algorithm, provided that the number of the voltage samples on each helix turn be always the same and equal to the smallest integer $M_{H}$, product of powers of 2, 3, and 5 equal or greater than $2\left[\operatorname{Int}\left(\chi^{\prime} \beta \rho_{\max }\right)+1\right], \rho_{\max }$ being the AUT maximum transverse dimension. In such a way, the samples lying on the helix at $\varphi_{m}=m \Delta \varphi=2 \pi m / M_{H}$ with $m=0, \ldots, M_{H}-1$ are all aligned. Accordingly, we get

$$
\begin{aligned}
& \int_{0}^{2 \pi} \tilde{V}^{1,2}\left(\vartheta_{n}, \varphi\right) G_{n \eta}(\varphi) e^{-j \nu \varphi} d \varphi \\
& \quad=\frac{2 \pi}{M_{H}} \sum_{m=0}^{M_{H}-1} \tilde{V}^{1,2}\left(\vartheta_{m, n}, \varphi_{m}\right) G_{n \eta}\left(\varphi_{m}\right) e^{-j 2 \pi m v / M_{H}},
\end{aligned}
$$

where

$$
\vartheta_{m, n}=\vartheta_{n}\left(\varphi_{m}\right)=\vartheta\left(\phi_{i}\right)+k \varphi_{m}+n \Delta \vartheta
$$

The summation in (17) can be, obviously, efficiently performed via a direct FFT algorithm.

It is worthy to note that the $G_{n \eta}\left(\varphi_{m}\right)$ values can be calculated (once and for all) for given sets of antennas.

According to the above results, the determination of the far field is obtained through the following steps.

(a) The samples of the probe and rotated probe voltages acquired at the constant step $\Delta \varphi$ along the scanning helix specified in Section 2 are multiplied for the phase factor $e^{j \gamma}$.

(b) For each required value of the polar angle $\Theta$, fixing the corresponding value of $\eta$ by the relation $\eta=$ $\beta \cos \Theta$, the $G_{n \eta}\left(\varphi_{m}\right)$ values are computed or read, if already calculated.

(c) For each helix turn, the FFT of the sequence $\tilde{V}^{1,2}\left(\vartheta_{m, n}, \varphi_{m}\right) G_{n \eta}\left(\varphi_{m}\right)$ is performed.

(d) The probe voltage Fourier transforms $I_{\nu}^{1,2}(\eta)$ are evaluated by performing the summation over $n \in$ $N_{r}$. The corresponding values of the cylindrical modal expansion coefficients $a_{v}$ and $b_{v}$ can be then evaluated.

(e) The evaluation of the far field at the considered angle $\Theta$ is finally effectively achieved by performing the summations in (13) again with the FFT.

From the efficiency viewpoint, it is convenient to use this method to evaluate only the FF samples necessary to recover the antenna pattern via the following far-field OSI expansion 
tailored for even numbers of samples along the meridians and parallels [10]:

$$
\begin{gathered}
F_{\Theta, \Phi}(\Theta, \Phi) \\
=\frac{2 N_{F}^{\prime \prime}-1}{2 N_{F}^{\prime \prime}} \sum_{n=n_{0}-q+1}^{n_{0}+q}\left\{\Omega_{N_{F}}\left(\Theta-\Theta_{n}\right) D_{N_{F}^{\prime \prime}-1}\left(\Theta-\Theta_{n}\right) \frac{2 M_{n}^{\prime \prime}-1}{2 M_{n}^{\prime \prime}}\right. \\
\times \sum_{m=m_{0}-p+1}^{m_{0}+p} F_{\Theta, \Phi}\left(\Theta_{n}, \Phi_{m, n}\right) \\
\times \Omega_{M n}\left(\Phi-\Phi_{m, n}\right) \\
\left.\times D_{M_{n}^{\prime \prime}-1}\left(\Phi-\Phi_{m, n}\right)\right\},
\end{gathered}
$$

wherein $n_{0}=\operatorname{Int}[\Theta / \Delta \Theta]$ and $m_{0}=\operatorname{Int}\left[\Phi / \Delta \Phi_{n}\right]$ are the indexes of the sample nearest (on the left) to the output point, and

$$
\begin{gathered}
\Theta_{n}=n \Delta \Theta=\frac{n \pi}{N_{F}^{\prime \prime}}, \quad \Phi_{m, n}=m \Delta \Phi_{n}=\frac{m \pi}{M_{n}^{\prime \prime}}, \\
N_{F}^{\prime \prime}=2\left[\operatorname{Int}\left(\frac{\chi N^{\prime}}{2}\right)+1\right], \quad N_{F}=N_{F}^{\prime \prime}-N^{\prime}, \\
M_{n}^{\prime \prime}=2^{i} \geq \operatorname{Int}\left(\chi M_{n}^{\prime}\right)+1, \quad M_{n}^{\prime}=\operatorname{Int}\left[\chi^{*} \beta \rho_{\max } \sin \Theta_{n}\right]+1, \\
M_{n}=M_{n}^{\prime \prime}-M_{n}^{\prime}, \quad \chi^{*}=1+\left(\chi^{\prime}-1\right)\left[\sin \Theta_{n}\right]^{-2 / 3} .
\end{gathered}
$$

The need of an OSI expansion tailored for an even number of samples along the parallels is due to the employment of an efficient power of two FFT algorithms for computing (13), whereas $N_{F}^{\prime \prime}$ has been chosen according to (21) in order to have FF samples on the equator.

It is worthy to note that there is no need to extract the phase factor from the far-field expression, since it is constant on the far-field sphere.

\section{Numerical and Experimental Results}

Many numerical tests have been performed to assess the validity of the presented technique. In particular, three sets of figures are reported.

The first one refers to a uniform planar circular array (see Figure 1) symmetric with respect to the plane $z=0$ and having radius $a=16 \lambda, \lambda$ being the wavelength. Its elements are elementary Huygens sources linearly polarized along the $z$-axis, radially and azimuthally spaced of $0.6 \lambda$. An open-ended WR-90 rectangular waveguide, operating at the frequency of $10 \mathrm{GHz}$, is considered as probe. The radius $d$ of the cylinder wrapped by the scanning helix is $25 \lambda$, and its height $h$ is $150 \lambda$.

Figures 2 and 3 show the FF pattern reconstruction in the principal planes obtained by using the direct helicoidal NFFF transformation described in Section 4. As can be seen, the exact and recovered fields are practically indistinguishable, thus assessing the effectiveness of the technique. Moreover, a

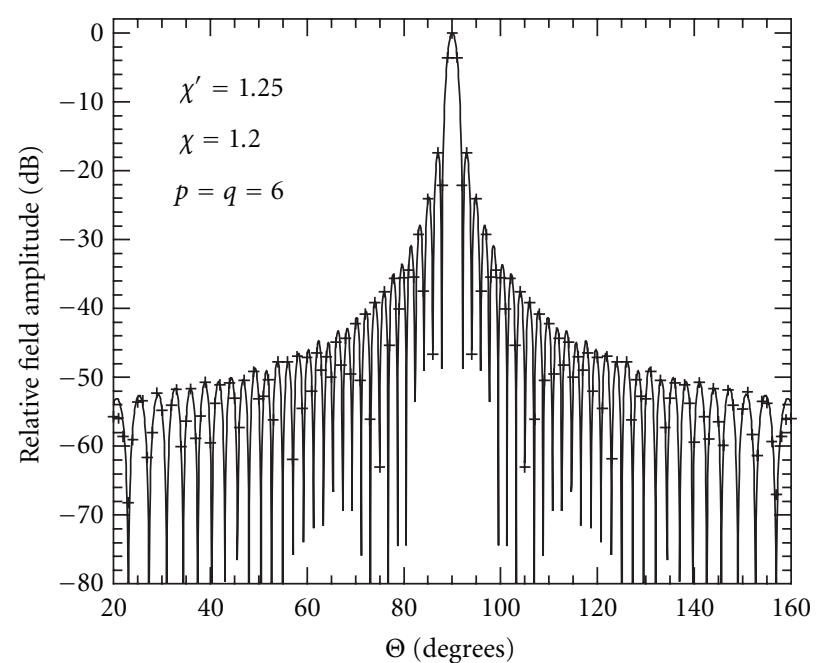

Figure 2: Far-field pattern in the E-plane. Solid line: exact field. Crosses: reconstructed via the direct helicoidal NF-FF transformation.

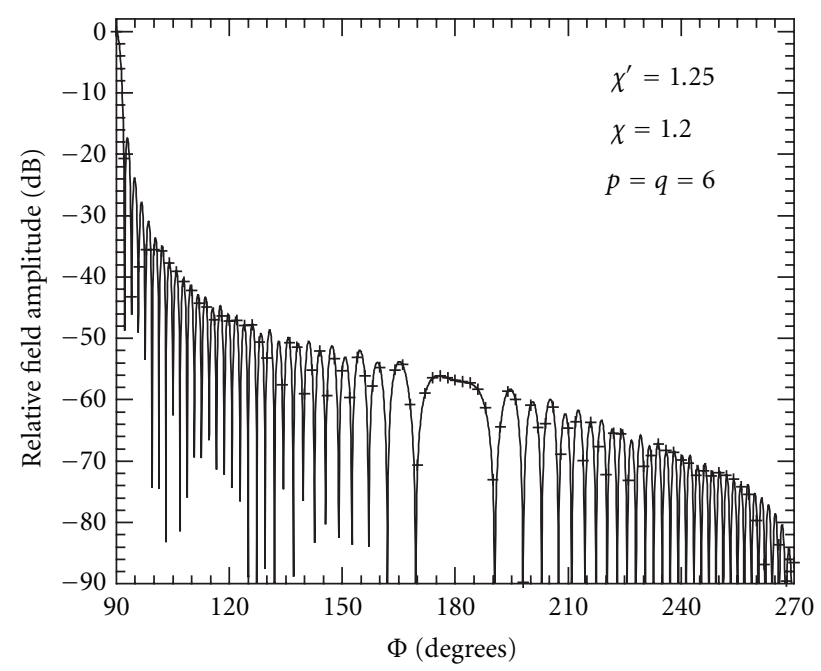

Figure 3: Far-field pattern in the $H$-plane. Solid line: exact field. Crosses: reconstructed via the direct helicoidal NF-FF transformation.

further example showing the reconstructed FF pattern in the plane at $\Phi=60^{\circ}$ is reported in Figure 4, in order to show the capability of the technique to accurately reconstruct the far field in any cut-plane.

The second set of figures is relevant to a smaller antenna. It is again a uniform planar circular array of elementary Huygens sources linearly polarized along the $z$-axis. Its radius $a$ is now $4.2 \lambda$, and its elements are radially and azimuthally spaced of $0.4 \lambda$. The scanning helix wraps a cylinder with height $h$ equal to $80 \lambda$ and radius $d=15 \lambda$. In order to highlight another peculiar feature of the here proposed direct helicoidal NF-FF transformation, the FF patterns in the $E$ plane obtained by applying such a NF-FF transformation and the standard cylindrical probe-compensated one [31] are compared with the exact FF pattern in Figures 5 and 6, 


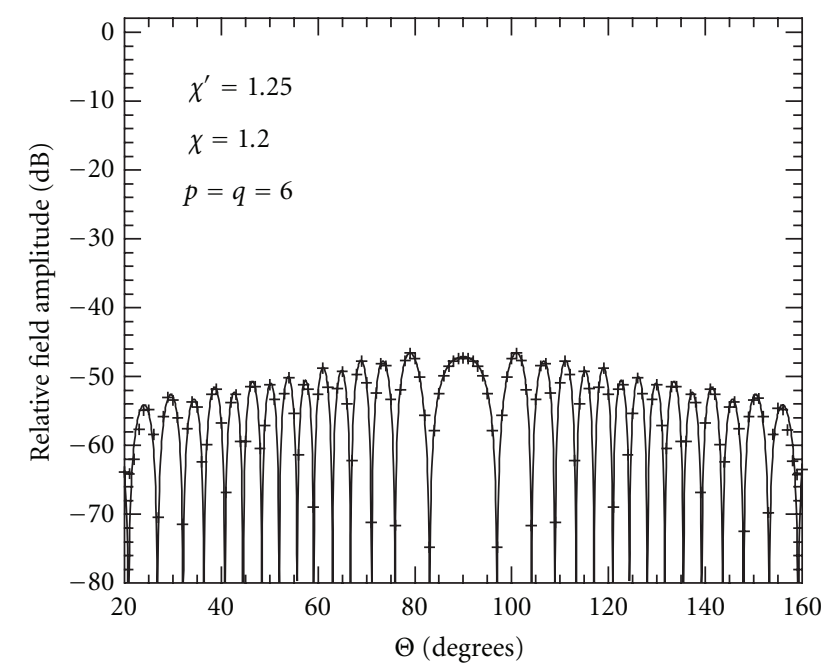

Figure 4: Far-field pattern in the plane at $\Phi=60^{\circ}$. Solid line: exact field. Crosses: reconstructed via the direct helicoidal NF-FF transformation.

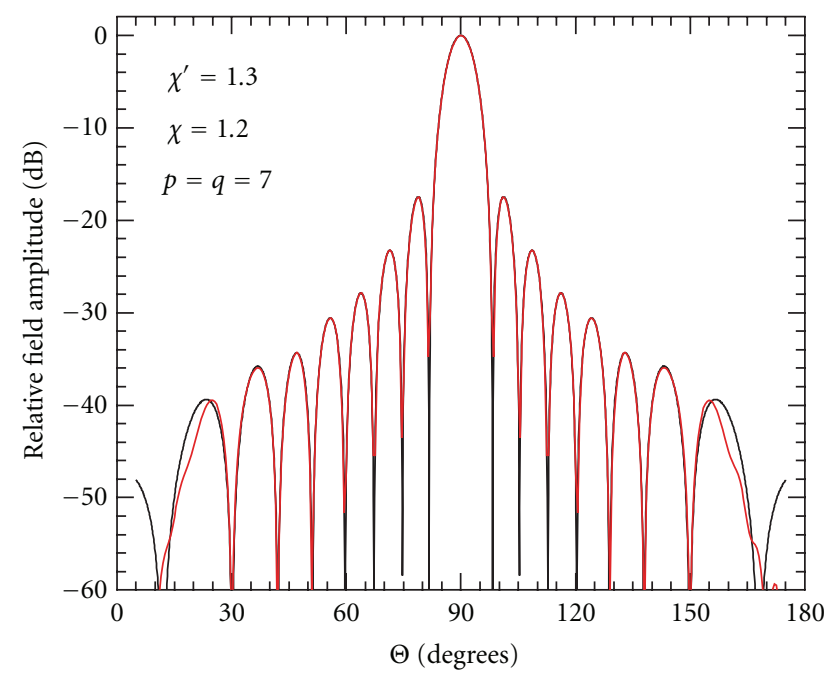

FIGURE 5: Far-field pattern in the E-plane. Black line: exact field. Red line: reconstructed via the direct helicoidal NF-FF transformation.

respectively. As can be seen, the reconstruction obtained via the proposed transformation is clearly more accurate than that achieved by the standard one, since the characteristic ripple caused by the discontinuity of the near field at the edge of the scanning surface is not present. This is due to the different method employed to evaluate the two-dimensional Fourier transforms $I_{v}^{1}$ and $I_{\nu}^{2}$ of the probe voltage. As a matter of fact, in the standard transformation technique [31], they are computed via FFT by taking into account only the NF data falling in the measurement area so that the integration over $z$ results truncated to the cylinder height. When the evaluation of $I_{\nu}^{1}$ and $I_{v}^{2}$ is carried out according to the proposed technique, the effect of each NF sample is considered (see (15)) in the range $\left[z_{i}, z_{f}\right]$, so that the peripheral samples can affect the evaluation even far from the scanning area. Thus, the proposed technique intrinsically

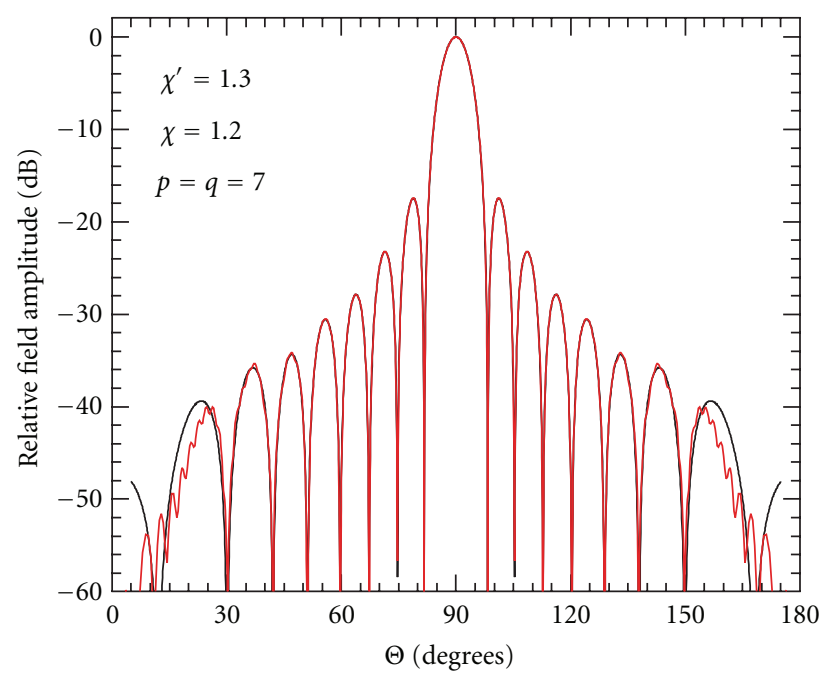

Figure 6: Far-field pattern in the E-plane. Black line: exact field. Red line: reconstructed via the standard cylindrical NF-FF transformation [31].

eliminates the field discontinuity at the edges of the scanning surface, without using any windowing function, that, as well known, reduces the ripple as well as the zone of good far-field reconstruction.

The third set of figures refers to the experimental validation of the direct helicoidal NF-FF transformation. Such a validation has been carried out in the anechoic chamber of the UNISA Antenna Characterization Lab. The chamber is equipped with a rotating table and a vertical scanner supplied by MI Technologies. The amplitude and phase measurements are performed by means of a vectorial network analyzer. An open-ended WR90 rectangular waveguide is used as probe. The considered antenna is a MI-12-8.2 standard gain horn with aperture $19.4 \mathrm{~cm} \times 14.4 \mathrm{~cm}$, located on the plane $y=0$ of the adopted reference system and operating at the frequency of $10 \mathrm{GHz}$. Such an AUT has been considered as enclosed in a spherical surface having radius equal to $12.6 \mathrm{~cm}$. The probe output voltages have been collected along a helix lying on cylinder with $d=45 \mathrm{~cm}$ and $h=240 \mathrm{~cm}$.

It must be stressed that the proposed NF-FF transformation incorporates the probe characterization. Therefore, first of all, the employed probe has been characterized according to [33] as done in the software package MI3000 implementing the standard probe compensated NFFF transformation with cylindrical scanning [32]. Then, it has been verified that practically identical results are obtained when the same NF data are transformed by using the MI package and the so developed version of the probe compensated NF-FF transformation [31]. Afterwards, the FF pattern in the principal planes $E$ and $H$ reconstructed by using the direct helicoidal NF-FF transformation has been compared with that obtained via the MI software (reference 1). These reconstructions are shown in Figures 7 and 8 . As can be seen, there is a good agreement save for the zones characterized by very low field levels, wherein the discrepancies are due to the fact that two different NF data 


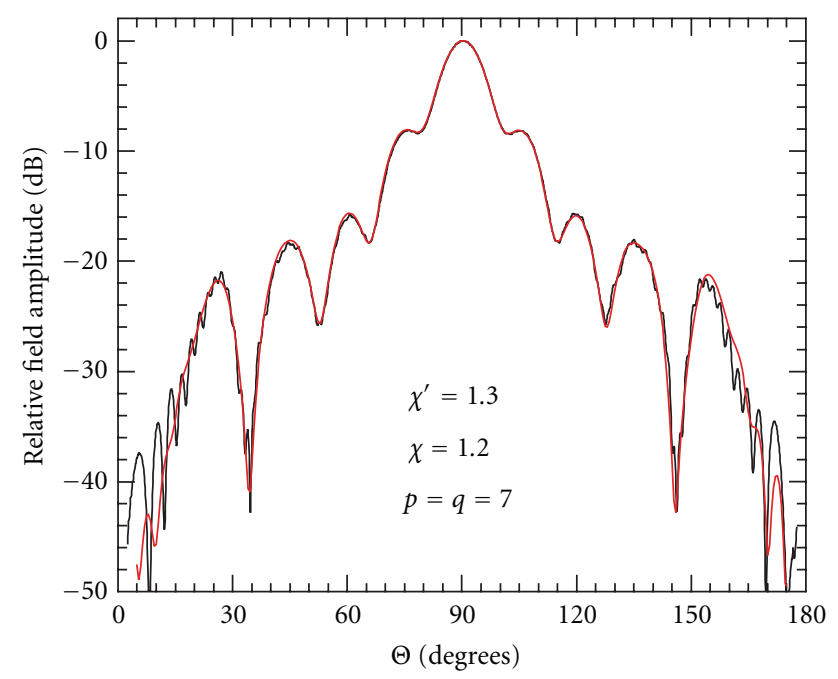

Figure 7: Far-field pattern in the E-plane. Black line: reference 1. Red line: reconstructed via the direct helicoidal NF-FF transformation.

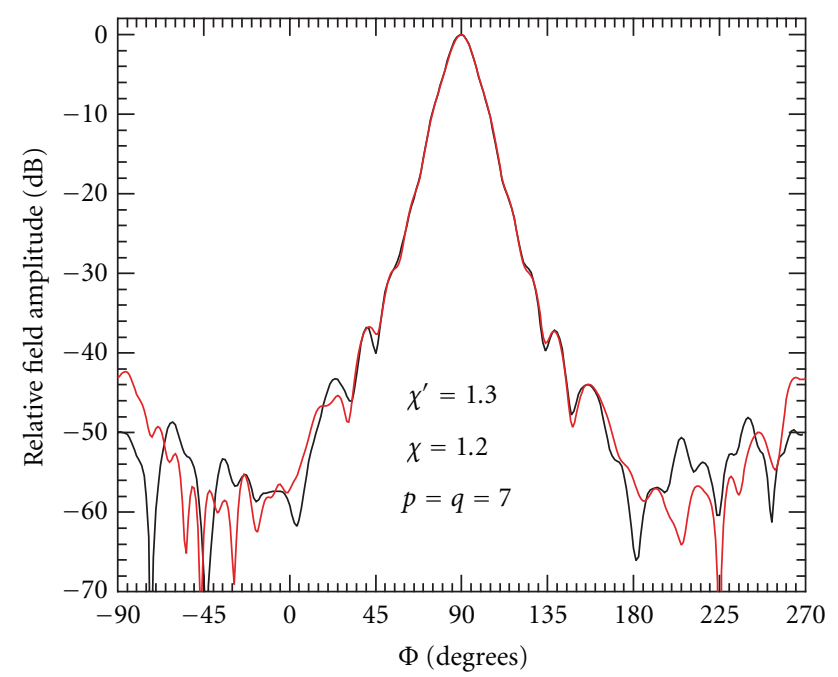

FIGURE 8: Far-field pattern in the $H$-plane. Black line: reference 1. Red line: reconstructed via the direct helicoidal NF-FF transformation.

sets have been employed. As expected, a better agreement results (see Figures 9 and 10) when the NF data used by the direct helicoidal NF-FF transformation are employed to obtain via a proper interpolation expansion those needed by the software package MI-3000, thus getting a new FF pattern (reference 2). The last reconstruction example (Figure 11) is relevant to the FF pattern in the plane at $\Phi=60^{\circ}$.

As can be seen, the experimental results relevant to the $\mathrm{FF}$ reconstructions in the $E$-plane and in the plane at $\Phi=60^{\circ}$ confirm the particular property of the developed technique to eliminate the ripple caused by the discontinuity of the near field at the edges of the scanning surface.

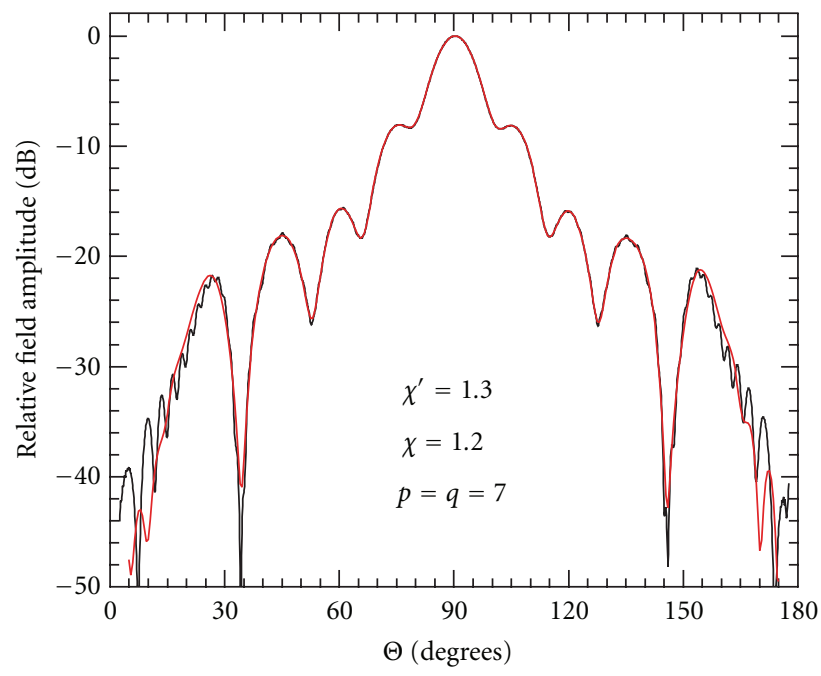

Figure 9: Far-field pattern in the E-plane. Black line: reference 2. Red line: reconstructed via the direct helicoidal NF-FF transformation.

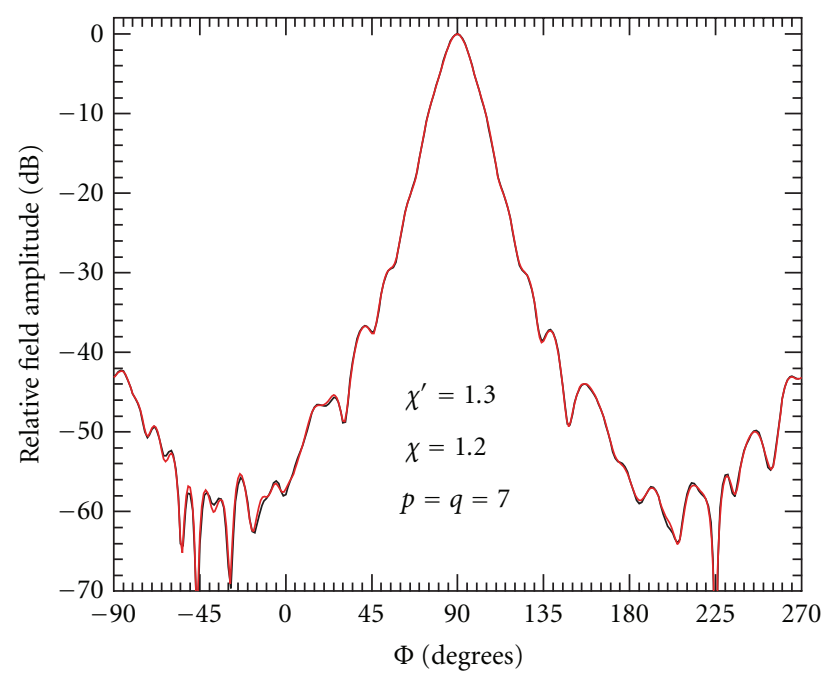

FIGURE 10: Far-field pattern in the $H$-plane. Black line: reference 2. Red line: reconstructed via the direct helicoidal NF-FF transformation.

It can be interesting to compare the number of NF data used by the direct NF-FF transformation (31 044 for the first example and 2417 for the other two) with those (67 725 for the first example and 11592 for the other two) needed by the MI software and by the helicoidal NF-FF transformation technique [30].

For what concerns the time needed for the NF data acquisition, the technique in [30] is certainly quicker than the traditional one, since the acquisition is performed by continuous and synchronized movements of the positioning systems. Accordingly, it is enough to compare the here developed technique only with the one in [30]. In such a case, 


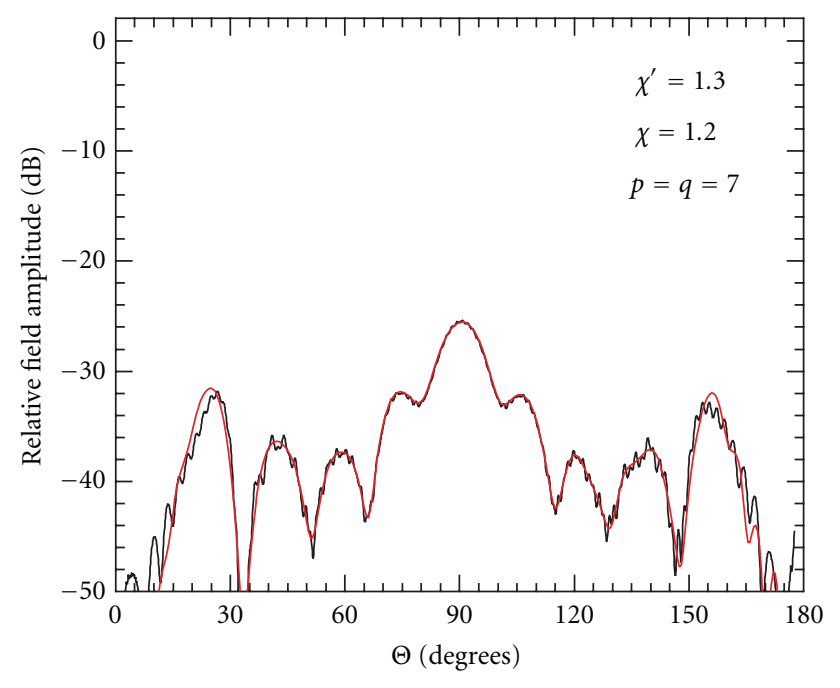

Figure 11: Far-field pattern in the plane at $\Phi=60^{\circ}$. Black line: reference 2. Red line: reconstructed via the direct helicoidal NF-FF transformation.

it can be easily realized that the measurement times are directly proportional to the numbers of needed samples.

\section{Conclusions}

A direct NF-FF transformation with helicoidal scanning, which allows one to evaluate the antenna far field in any cut plane directly from a minimum set of NF data without interpolating them, has been developed in this paper. To this end, the approach in [10] has been reviewed in order to match the advantages of the direct cylindrical NF-FF transformation with those own of the fast helicoidal scanning. In particular, the NF sampling arrangement is obtained by determining the number of helix turns according to the nonredundant sampling representation along a generatrix which makes use of the spherical AUT modelling and the voltage samples on each of them according to the AUT maximum transverse dimension. Many numerical tests have assessed the validity of the presented technique and its capability to eliminate the ripple caused by the discontinuity of the near field at the edges of the scanning surface. Moreover, the technique effectiveness has been further confirmed by the experimental results. At last, it has been shown that, although the number of NF data employed by the developed technique is slightly increased with respect to that required by rigorously applying the nonredundant sampling representation on the helix, it remains still remarkably smaller than that needed by the standard NF-FF transformation with cylindrical scan. It is worthy to note that the proposed technique, as well as all those using the spiral scannings, allow also the frequency extension of existing near-field ranges, since both measurement axes are used simultaneously hence reducing the effect of backlash.

\section{References}

[1] "Special Issue on near-field scanning techniques," IEEE Transactions on Antennas and Propagation, vol. AP-36, no. 6, pp. 727-901, 1988.

[2] A. D. Yaghjian, "An overview of near-field antenna measurements," IEEE Transactions on Antennas and Propagation, vol. AP-34, no. 1, pp. 30-45, 1986.

[3] C. Gennarelli, G. Riccio, F. D'Agostino, and F. Ferrara, NearField-Far-Field Transformation Techniques, vol. 1, CUES, Salerno, Italy, 2004.

[4] D. Slater, Near-Field Antenna Measurements, Artech House, Boston, Mass, USA, 1991.

[5] C. A. Balanis, Modern Antenna Handbook, John Wiley \& Sons, Hoboken, NJ, USA, 2008.

[6] O. M. Bucci, C. Gennarelli, G. Riccio, and C. Savarese, "Nearfield-far-field transformation from nonredundant planepolar data: effective modellings of the source," IEE Proceedings on Microwaves, Antennas and Propagation, vol. 145, no. 1, pp. 33-38, 1998.

[7] O. M. Bucci, G. D’Elia, and M. D. Migliore, "Advanced field interpolation from plane-polar samples: experimental verification," IEEE Transactions on Antennas and Propagation, vol. 46, no. 2, pp. 204-210, 1998.

[8] O. M. Bucci, F. D’Agostino, C. Gennarelli, G. Riccio, and C. Savarese, "NF-FF transformation with plane-polar scanning: ellipsoidal modelling of the antenna," Automatika, vol. 41, no. 3-4, pp. 159-164, 2000.

[9] F. D’Agostino, C. Gennarelli, G. Riccio, and C. Savarese, "Data reduction in the NF-FF transformation with bi-polar scanning," Microwave and Optical Technology Letters, vol. 36, no. 1, pp. 32-36, 2003.

[10] O. M. Bucci, C. Gennarelli, G. Riccio, V. Speranza, and C. Savarese, "Nonredundant representation of the electromagnetic fields over a cylinder with application to the near-field far-field transformation," Electromagnetics, vol. 16, no. 3, pp. 273-290, 1996.

[11] O. M. Bucci, C. Gennarelli, G. Riccio, and C. Savarese, "NFFF transformation with cylindrical scanning: an effective technique for elongated antennas," IEE Proceedings on Microwaves, Antennas and Propagation, vol. 145, no. 5, pp. 369-374, 1998.

[12] F. D’Agostino, F. Ferrara, C. Gennarelli, G. Riccio, and C. Savarese, "NF-FF transformation with cylindrical scanning from a minimum number of data," Microwave and Optical Technology Letters, vol. 35, no. 4, pp. 264-268, 2002.

[13] O. M. Bucci, F. D’Agostino, C. Gennarelli, G. Riccio, and C. Savarese, "Data reduction in the NF-FF transformation technique with spherical scanning," Journal of Electromagnetic Waves and Applications, vol. 15, no. 6, pp. 755-775, 2001.

[14] F. D’Agostino, F. Ferrara, C. Gennarelli, R. Guerriero, and M. Migliozzi, "Effective antenna modellings for NF-FF transformations with spherical scanning using the minimum number of data," International Journal of Antennas and Propagation, vol. 2011, Article ID 936781, 11 pages, 2011.

[15] O. M. Bucci, C. Gennarelli, and C. Savarese, "Representation of electromagnetic fields over arbitrary surfaces by a finite and nonredundant number of samples," IEEE Transactions on Antennas and Propagation, vol. 46, no. 3, pp. 351-359, 1998.

[16] O. M. Bucci, C. Gennarelli, and C. Savarese, "Fast and accurate near-field-far-field transformation by sampling interpolation of plane-polar measurements," IEEE Transactions on Antennas and Propagation, vol. 39, no. 1, pp. 48-55, 1991. 
[17] R. G. Yaccarino, L. I. Williams, and Y. Rahmat-Samii, "Linear spiral sampling for the bipolar planar near-field antenna measurement technique," IEEE Transactions on Antennas and Propagation, vol. 44, no. 7, pp. 1049-1051, 1996.

[18] O. M. Bucci, C. Gennarelli, G. Riccio, and C. Savarese, "Nonredundant NF-FF transformation with helicoidal scanning," Journal of Electromagnetic Waves and Applications, vol. 15, no. 11, pp. 1507-1519, 2001.

[19] F. D’Agostino, F. Ferrara, C. Gennarelli, R. Guerriero, and M. Migliozzi, "Experimental results validating the near-field to far-field transformation technique with helicoidal scan," The Open Electrical \& Electronic Engineering Journal, vol. 4, pp. 1015, 2010.

[20] F. D’Agostino, C. Gennarelli, G. Riccio, and C. Savarese, "Theoretical foundations of near-field-far-field transformations with spiral scannings," Progress in Electromagnetics Research, vol. 61, pp. 193-214, 2006.

[21] O. M. Bucci, F. D’Agostino, C. Gennarelli, G. Riccio, and C. Savarese, "Probe compensated far-field reconstruction by near-field planar spiral scanning," IEE Proceedings: Microwaves, Antennas and Propagation, vol. 149, no. 2, pp. 119-123, 2002.

[22] O. M. Bucci, F. D’Agostino, C. Gennarelli, G. Riccio, and C. Savarese, "Near-field-far-field transformation with spherical spiral scanning," IEEE Antennas and Wireless Propagation Letters, vol. 2, pp. 263-266, 2003.

[23] F. D'Agostino, F. Ferrara, C. Gennarelli, R. Guerriero, and M. Migliozzi, "Near-field-far-field transformation technique with helicoidal scanning for elongated antennas," Progress in Electromagnetics Research B, vol. 4, pp. 249-261, 2008.

[24] F. D’Agostino, F. Ferrara, J. A. Fordham et al., "An effective near-field-far-field transformation technique for elongated antennas using a fast helicoidal scan," IEEE Antennas and Propagation Magazine, vol. 51, no. 4, Article ID 5338700, pp. 134-141, 2009.

[25] F. D’Agostino, F. Ferrara, C. Gennarelli, R. Guerriero, and M. Migliozzi, "Laboratory tests assessing the effectiveness of the NF-FF transformation with helicoidal scanning for electrically long antennas," Progress in Electromagnetics Research, vol. 98, pp. 375-388, 2009.

[26] F. D’Agostino, F. Ferrara, C. Gennarelli, R. Guerriero, and M. Migliozzi, "An effective NF-FF transformation technique with planar spiral scanning tailored for quasi-planar antennas," IEEE Transactions on Antennas and Propagation, vol. 56, no. 9, pp. 2981-2987, 2008.

[27] F. D’Agostino, F. Ferrara, C. Gennarelli, R. Guerriero, M. Migliozzi, and G. Riccio, "A nonredundant near-field to far-field transformation with spherical spiral scanning for nonspherical antennas," The Open Electrical \& Electronic Engineering Journal, vol. 3, pp. 1-8, 2009.

[28] F. D’Agostino, F. Ferrara, C. Gennarelli, R. Guerriero, and M. Migliozzi, "The unified theory of near-field-far-field transformations with spiral scannings for nonspherical antennas," Progress In Electromagnetics Research B, no. 14, pp. 449-477, 2009.

[29] S. Costanzo and G. Di Massa, "Near-field to far-field transformation with planar spiral scanning," Progress in Electromagnetics Research, vol. 73, pp. 49-59, 2007.

[30] S. Costanzo and G. Di Massa, "Far-field reconstruction from phaseless near-field data on a cylindrical helix," Journal of Electromagnetic Waves and Applications, vol. 18, no. 8, pp. 1057-1071, 2004.
[31] W. M. Leach and D. T. Paris, "Probe compensated near-field measurements on a cylinder," IEEE Transactions on Antennas and Propagation, vol. AP-21, no. 4, pp. 435-445, 1973.

[32] A. D. Yaghjian, "Near-field antenna measurement on a cylindrical surface: a source scattering matrix formulation," NBS Technical Note 696, U.S. Government Printing Office, Washington, DC, USA, 1977.

[33] A. D. Yaghjian, "Approximate formulas for the far field and gain of open-ended rectangular waveguide," IEEE Transactions on Antennas and Propagation, vol. AP-32, no. 4, pp. 378-384, 1984. 

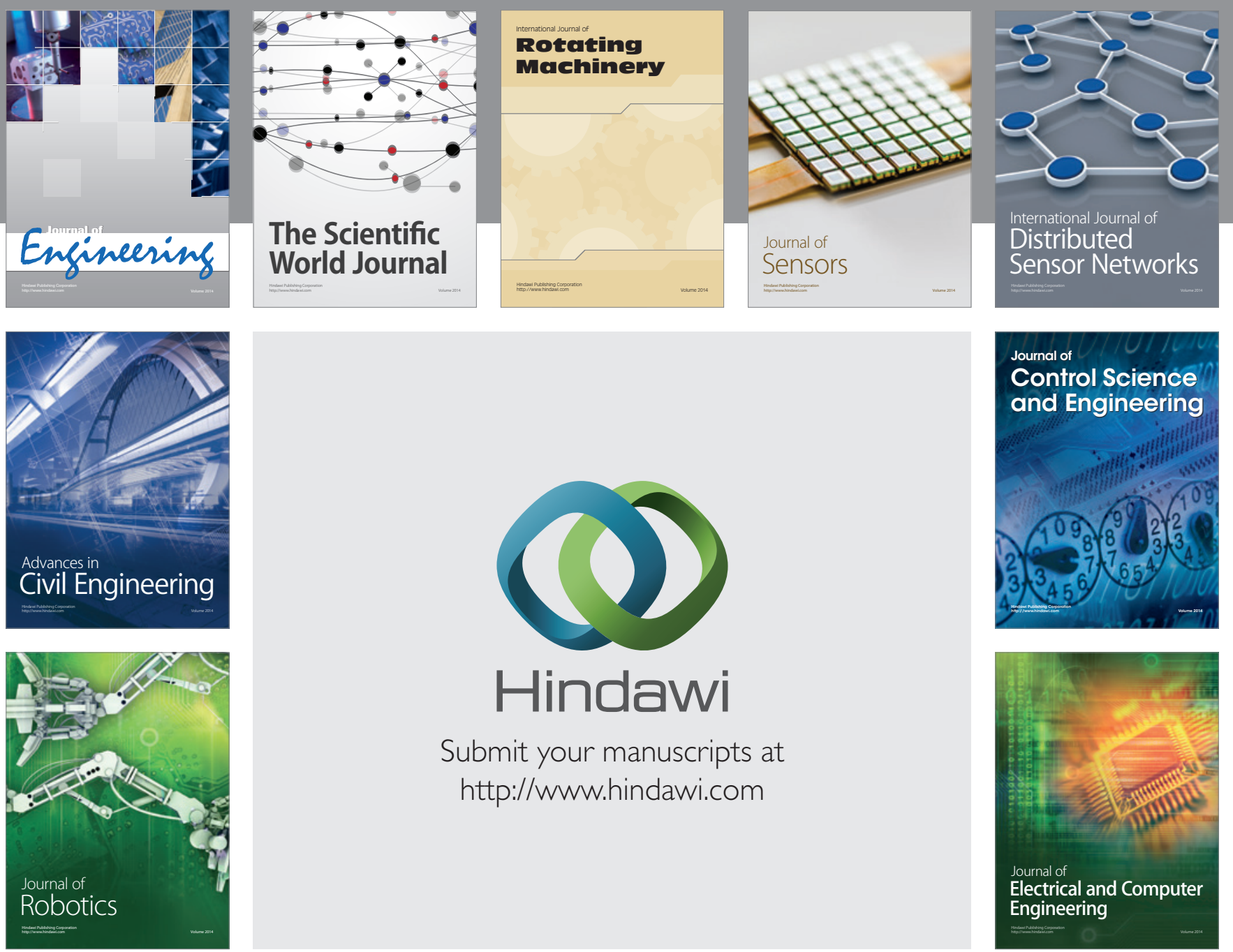

Submit your manuscripts at

http://www.hindawi.com
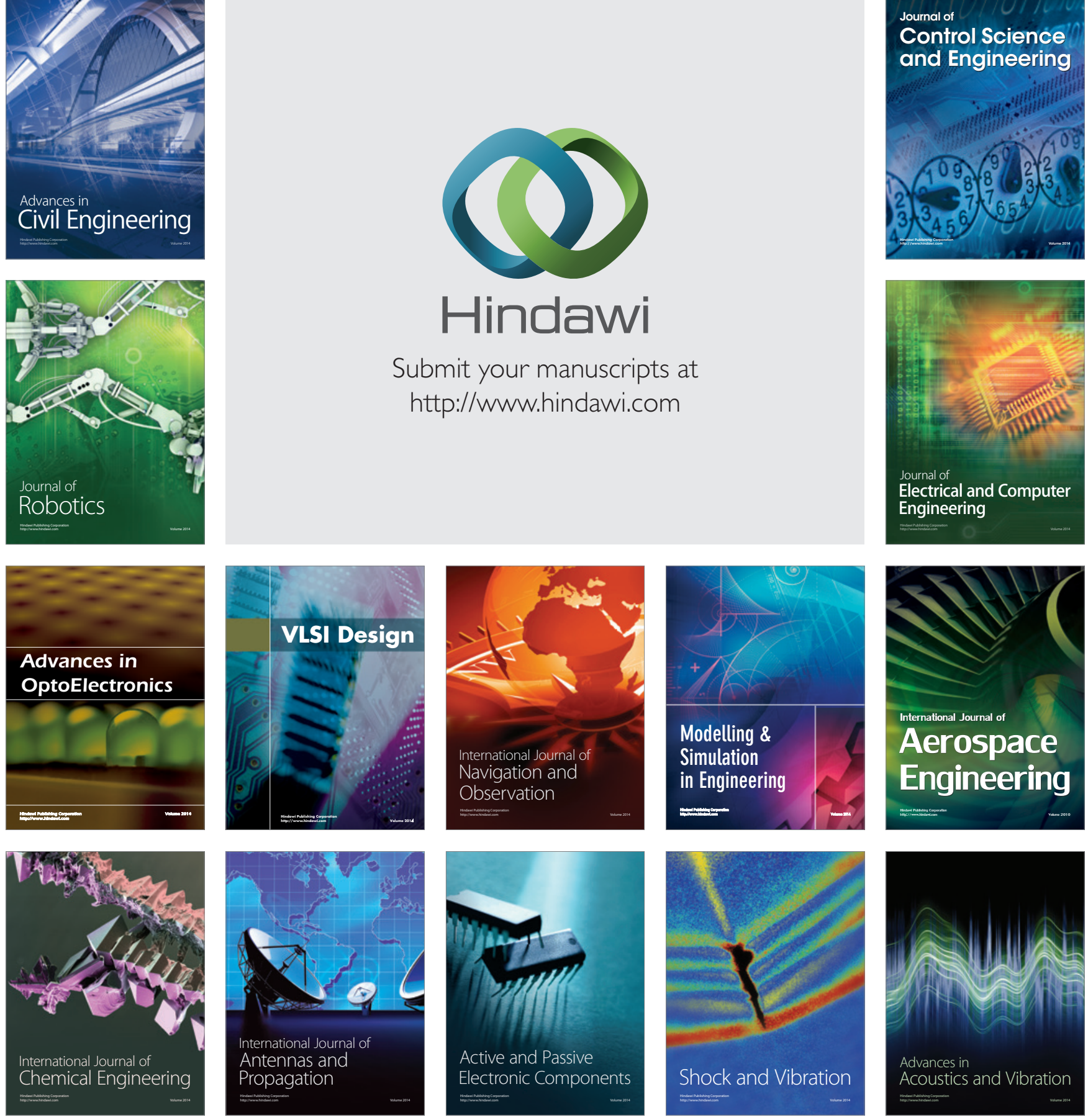